\title{
ANDERSEN-TAWIL SYNDROME IN A NIGERIAN WOMAN: A CASE REPORT AND REVIEW OF LITERATURE
}

\author{
*Owolabi LF, *Iliyasu G, *Shehu MN, *Ibrahim A. *Shehu MY \\ * Department Of Medicine, Aminu Kano Teaching Hospital, Kano, Nigeria.
}

\begin{abstract}
.
The case is a 35-year-old woman with recurrent spontaneous weakness of all the limbs of 2-year duration she had had four episodes in the past, the last 2 episodes were during pregnancy and immediate post natal period respectively, each episode lasted for five to ten days. There was a close consanguinity between her parents (parents are cousins). She also has an elder sister with recurrent paraplegia.

On physical examination she weighed $80 \mathrm{~kg}$ and her height was $156 \mathrm{~cm}$, she had low-set ears, high arched palate, brachydactylyl, and bilateral pedal lymphoedema. Muscle power was grade 1 in all the limb at presentation, the tone was reduced globally; the deep tendon reflexes were normoactive in all the limbs. On laboratory investigation, serum potassium was consistently low during all the episodes $(2 \mathrm{meq} / \mathrm{L}$, $1.5 \mathrm{meq} / \mathrm{L}, 1.7 \mathrm{meq} / \mathrm{L}$ and $1.5 \mathrm{meq} / \mathrm{L}$ respectively), on electrocardiogram, arrhythmia was not present, but QTc interval was 0.46 seconds. Based on the clinical and laboratory abnormalities she was diagnosed with Anderson Tawil Syndrome. She was given potassium supplementation and acetazolamide and remarkable improvement was recorded in her symptoms.
\end{abstract}

Keywords: Andersen-Tawil syndrome, quadriplegia, syncope, prolonged QTc interval.

\section{Introduction}

In 1963, two cases of familial periodic paralysis associated with ventricular ectopy were first described $^{1}$, the suggestion of a potential syndromic association was not proposed until 1971, when Ellen Andersen and colleagues described the characteristic triad in an 8 year-old dysmorphic boy who experienced episodes of muscle weakness and ventricular extrasystoles ${ }^{2}$.

Subsequently, the syndrome was named AndersenTawil Syndrome (ATS) ${ }^{3}$. In 2001, mutations in KCNJ2, which encodes the $a$-subunit of the potassium channel Kir2.1, were identified in patients with ATS. To date, KCNJ2 is the only gene implicated in ATS, accounting for approximately $60 \%$ of cases. ATS is a unique channelopathy, and represents the first link between cardiac and skeletal muscle excitability.

AndersenTawil Syndrome (ATS) is a heterogeneous autosomal dominant or sporadic disorder characterized by the clinical triad of periodic paralysis, dysmorphic features, and cardiac arrhythmias. ${ }^{4,5}$. Periodic paralyses are rare inherited channelopathies that manifest as abnormal, often potassium sensitive, muscle membrane excitability leading to episodic flaccid paralysis, hypokalaemic and hyperkalemic periodic paralyses could occur in Andersen-Tawil syndrome (ATS) ${ }^{4,6-8}$. A little above 100 cases of Anderson Tawil Syndrome have been reported worldwide. In this article, we report a Nigerian woman with probable Anderson-Tawil Syndrome because of the rarity of this disorder.

\section{Case report}

The case is a 35-year-old woman with recurrent spontaneous weakness of all the limbs of 2-year duration she had had four episodes in the past, the last 2 episodes were during pregnancy and immediate post natal period respectively, each episode lasted for five to ten days. The episode she had during pregnancy occurred at 24 weeks of gestation. She also had 2 episodes of syncopal attack, each lasting about 2 minutes, No jerky movement of the extremities, deviation of the eyes, urinary incontinence, and cyanosis was noted during the syncope attacks. No associated loss of sensation to touch and pain during the quadriplegia attacks. The last quadriplegic attack started two days before admission to the hospital. She was previously diagnosed with Gullain Barre syndrome but no improvement was noted on treatment. Her prenatal, natal and postnatal history was uneventful. There was a close consanguinity between her parents (parents are cousins). After careful evaluation, we learnt that she had an elder sister with recurrent paraplegia who, to the best of her knowledge, has had two attacks.

On physical examination she weighed $80 \mathrm{~kg}$ and her height was $156 \mathrm{~cm}$, she had low-set ears, high arched palate, brachydactylyl, and bilateral pedal lymphoedema. Muscle power was grade 1 in all the limbs at presentation, the tone was reduced globally; the deep tendon reflexes were normoactive in all the limbs. There was no impairment of sensation to touch and pain stimuli, joint position sense and vibration sense were intact. She also had infrequent missed heart beat otherwise 
the remainder of the physical examination was unremarkable.

On laboratory investigation, serum potassium was consistently low during all the episodes $(2 \mathrm{meq} / \mathrm{L}$, $1.5 \mathrm{meq} / \mathrm{L}, 1.7 \mathrm{meq} / \mathrm{L}$ and $1.5 \mathrm{meq} / \mathrm{L}$ respectively), other serum electrolytes levels were within normal limits. Complete blood count, renal and liver function tests were normal. At admission, Microscopic examination of urine was unremarkable. Abdominal ultrasonography was normal. On electrocardiogram, arrhythmia was not present, but Q Tc interval was 0.46 seconds. However genetic study was not done due to lack of facilities to carry it out.

On the $3^{\text {rd }}$ day of admission her quadriplegia completely resolved on potassium supplementation, repeat serum potassium was $4.3 \mathrm{meq} / \mathrm{L}$ at that time. Based on the clinical and laboratory abnormalities she was diagnosed with Anderson Tawil Syndrome. On the $7^{\text {th }}$ hospital day she was discharged from the hospital with potassium supplementation and acetazolamide and she is currently been followed up.

\section{Discussion}

Anderson Tawil Syndrome is an uncommon autosomal dominant disorder characterized by periodic paralysis, cardiac arrhythmias, and dysmorphic features ${ }^{4,9,10}$. In 1971, Andersen et al, ${ }^{2}$ described a boy with low-set ears, hypertelorism, broad nasal root, mandibular hypoplasia, soft and hard palate defects, scaphocephaly, clinodactyly, short stature, extrasystoles, and attacks or muscular weakness. Tawil et al, ${ }^{11}$ first named this entity Andersen syndrome and proposed a clinical triad including some minor anomalies, cardiac arrhythmia, and potassium sensitive periodic paralysis as the criteria for establishing the diagnosis of this disorder. The cardiac involvement in this syndrome seems to be different from that in other entities that can cause arrhythmias and syncope, such as the long QT syndrome in which the cardiac abnormalities are related to an abnormal function of ion channels ${ }^{12}$. Canum et al. ${ }^{9}$ reported on ATS a three-generation family with 10 affected members. They noted some additional minor anomalies such as broad forehead and malar hypoplasia. Their patients had variable expression in the classical triad and of the severity of the systemic manifestations. Five of eight affected studied members did not have a long QTc, which has been suggested as a constant finding in this syndrome $^{9}$
As regard the diagnosis of this disorder, appreciation of dysmorphic manifestations constitute one of the three fundamental elements in the evaluation of ATS. Features initially described included short stature, hypertelorism (wide-set eyes), low-set ears, palatal defects, mandibular hypoplasia, single palmar crease, crytporchidism, and slight bilateral ptosis ${ }^{2}$. In a recent series of 36 KCNJ2 mutation carriers, $78 \%$ had at least two dysmorphic features, the most common being clinodactyly (permanent medial or lateral curvature of a finger or toe, seen in 64\%) and mandibular hypoplasia $(44 \%)^{5}$ Other features described have included scoliosis, hyperthyroidism, vaginal atresia, and unilateral dysplastic kidney; structural cardiovascular anomalies such as a bicuspid aortic valve with or without associated coarctation, and valvular pulmonic stenosis have been reported as well ${ }^{13}$. Also noted in one series was that the severity of facial dysmorphism did not correlate with the severity of cardiac or skeletal muscular involvement ${ }^{9}$.

The cardiac manifestation show variability in phenotypic expression, there exists a wide range of electrophysiologic manifestations in ATS. Some patients have QT interval prolongation, while others demonstrate normal QT intervals. Prominent U waves in the anterior precordial leads are common in $\mathrm{ATS}^{14}$. Although $\mathrm{U}$ waves can be observed in normal individuals at low heart rates or in hypokalemic states, these are described in ATS patients at higher heart rates, suggesting that this may represent a manifestation of disease rather than a normal variant ${ }^{5}$. Associated arrhythmias range from isolated premature ventricular beats to complex ventricular ectopy and polymorphic ventricular tachycardias such as bidirectional ventricular tachycardia or less commonly torsades depointes.

In 2001, Plaster et al, ${ }^{4}$ mapped an Andersen's locus to chromosome $17 \mathrm{q} 23$ near the inward rectifying potassium channel gene KCNJ2. A missense mutation in KCNJ2 (encoding D7IV) was identified in the linked family. Eight additional mutations were identified in unrelated patients. Expression of two of these mutations in Xenopus oocytes revealed loss of function and a dominant negative effect in Kir2,1 current as assayed by voltage-clamp. They suggest that Kir2.1plays an important role in developmental signaling in addition to its previously recognized function in controlling cell excitability in skeletal muscle and heart ${ }^{4}$. However, it has been reported that over the past decade 
mutations in genes encoding three ion channels, CACNIAS, SCN4A and KCNJ2, have been identified and account for at least $70 \%$ of the identified cases of Periodic paralysis and several allelic disorders. No prospective clinical studies have followed sufficiently large cohorts with characterized molecular lesions to draw precise conclusions ${ }^{6}$.

In making a diagnosis of ATS, given the heterogenous nature of the clinical manifestations of this syndrome, a definitive diagnosis at times is elusive. Manifestation of at least two phenotypic classes (skeletal, cardiac, or developmental) has been thought to be necessary for diagnosis of ATS [5]. While some patients may manifest aspects consistent with all three classes of the clinical anomalies, others, (including those with known KCNJ2 mutations) may manifest only a single phenotypic classification. In these instances, some authors have suggested diagnosing such patients should they have a substantial family history ${ }^{3}$. Noting that to date, about $60 \%$ of Andersen syndrome patients are found to have mutations in Kir2.1, screening for $\mathrm{KCNJ} 2$ mutations also provides an increasingly valuable tool in either confirming or establishing diagnosis in a phenotypically heterogenous condition.

Our patient had recurrent quadriplegia attacks, dysmorphic features and prolonged QTc interval (0.46 seconds) and syncopal attacks. Her elder sister also had recurrent paraparesiss, however, she was not available for clinical, electrocardiographic and laboratory evaluation. Even though genetic study could not be done on this patient for lack of facilities, the presence of parental consanguinity and similar illness will strongly support the genetic basis of this disorder.

Therapy for ATS is directed toward its phenotypic manifestations which lead to significant morbidity, namely associated episodic weakness and most importantly its association with life-threatening ventricular tachyarrhythmias. From initial reports, therapies such as oral potassium supplementation, sodium restriction, spironolactone, and acetazolamide have anecdotally been shown to ameliorate symptoms of weakness ${ }^{4,9,11,15}$. We have observed improvement with oral contraceptive therapy, with exacerbation of weakness after discontinuation in a female patient with ATS. Unfortunately, perhaps due to the genotypic and phenotypic heterogeneity in disease, in concert with erratic and paradoxical exacerbation of symptoms with therapy, no therapeutic standards exist to date.
While the cardiac sequelae of ATS hold the greatest potential for morbidity and mortality, they remain the most problematic in devising effective medical therapeutic options.

Given the phenotypic heterogeneity that complicates diagnosis, this variability also plays a role as a complicating factor in effective risk stratification.

Our patient was given potassium supplementation and acetazolamide and remarkable improvement was recorded in her symptoms.

\section{References}

1. Klein R, Ganelin R, Marks JF, Usher P, Richards C. J Periodic paralysis with cardiac arrhythmia. J Pediatr. 1963; 62:371-85.

2. Andersen ED, Krasilnikoff PA, Overvad H. Intermittent muscular weakness, extrasystoles, and multiple developmental anomalies. A new syndrome? Acta Paediatr Scand. 1971; 60:559-64.

3. Donaldson MR, Jensen JL, Tristani-Firouzi M, Tawil R, Bendahhou S, Suarez WA, Cobo AM, Poza JJ, Behr E, Wagstaff J, Szepetowski P, Pereira S, Mozaffar T, Escolar DM, Fu YH, Ptacek LJ. PIP2 binding residues of Kir2.1 are common targets of mutations causing Andersen syndrome. Neurology. 2003; 60:1811-6.

4. Plaster NM, Tawil R, Tristani-Firouzi M, Canun S, Bendahhou S, Tsunoda A et al. Mutations in Kir2.1 cause the developmental and episodic electrical phenotypes of Andersen's syndrome. . Cell 2001; 105:51-9.

5. Tristani-Firouzi M Jensen JJ, Donaldson MR, Sansone V, Meola G, Hahn A, Bendahhou S, Kwiecinski H FA, Plaster N, Fu YH, Ptacek LJ, Tawil R. . Functional and clinical characterization of $\mathrm{KCNJ} 2$ mutations associated with LQT7 (Andersen syndrome). . J Clin Invest 2002; 110:381-8.

6. Venance SL,Canon SC, Failho D, Fontaine B, Hanna MG, Ptacek LJ et al. The primary periodic paralyses: diagnosis, pathogenesis and treatment. Brain 2006; 129:8-17.

7. Chun TU, Epstein MR, Dick M, Andelfinger G, Ballester L, Vanoye CG, George AL, Jr., Benson DW.

8. Preisig-Muller R, Sschlichthorl G, Goerge $\mathrm{T}$ et al. Heteromerization of Kir2.x potassium channels contributes to the phenotype of Andersen's syndrome, Proc Natl Acad Sci USA 2002;99:1774 7779.

9. Canun S, Perez N, Beirana LG. Andersen syndrome autosomal dominant in three generations. Am J Med Genet 1999; 85:147-56.

10. Sansone V, Griggs RC, Meola G et al. Andersen's syndrome: a distinct periodic paralysis. . Ann Neurol 1997; 42:305-12.

11. Tawil R, Tawil PL, Pavlakis SG, DeVivo DC, Penn AS, Ozdemir C, Griggs RC. Andersen's syndrome: 
potassium-sensitive periodic paralysis, ventricular ectopy, and dysmorphic features. Ann Neurol. 1994; 35:326-30.

12. Ackerman MJ, Clapham DE. Ion channels basic science and clinical disease, N Engl J Med 1997;336: 1375586.

13. Andelfinger G, Tapper A, Welch RC, Vanoye CG, George AL, Jr., Benson DW. KCNJ2 mutation results in Andersen syndrome with sex-specific cardiac and skeletal muscle phenotypes. Am J Hum Genet 2002; 71:663-8.

14. Zhang L BD, Tristani-Firouzi M, Ptacek LJ, Tawil R, Schwartz PJ, George AL, Horie M AG, Snow GL, Fu YH, Ackerman MJ, Vincent GM. Electrocardiographic features in Andersen-Tawil syndrome patients with KCNJ2 mutations: characteristic T-U-wave patterns predict the KCNJ2 genotype. . Circulation 2005; 111:2720-6.

15. Junker J, Haverkamp W, Schulze-Bahr E, Eckardt L, Paulus W, Kiefer R. . Amiodarone and acetazolamide for the treatment of genetically confirmed severe Andersen syndrome. . Neurology 2002; 59:466

Declaration.

Case report was presented at Nigerian Society of Neurological Sciences conference in Ibadan, 2008. 